\title{
THE COBORDISM CLASSIFICATION OF HYPERSURFACES IN LENS SPACES
}

\section{J. H. C. CREIGHTON}

\begin{abstract}
In Theorem A we classify by cobordism type the codimension one submanifolds of lens spaces $L_{d}^{2 n+1}$ (quotient of $S^{2 n+1}$ by the action of the $d$ th roots of unity). A related immersion result is also obtained.
\end{abstract}

Introduction. A classical generalization of the Jordon Curve Theorem asserts that every compact codimension one submanifold $M$ of the $n$-sphere $S^{n}$ separates. Further, since $S^{n}$ is orientable, $M$ is orientable. However, the sphere is not unique with this property, for if $N$ is an orientable manifold such that $H^{1}\left(N ; Z_{2}\right)=0$ then any codimenison one submanifold separates and is orientable.

We study the situation for more general ambient manifolds in this paper and obtain in Theorem A the cobordism classification of codimension one submanifolds in lens spaces, partially generalizing results of Bredon and Wood for 3-dimensional lens spaces. The basic technique will apply to other classes of ambient manifolds as well. A partial extension to codimension one immersions is also given. In $\$ 1$ we obtain some general results and state Theorem $A$, the proof of which is given in $\$ 2$.

I wish to thank Benjamin Halpern and W. D. Neumann for helpful comments on preliminary versions of this paper. I also thank the referee for his careful reading and for several improvements in exposition.

1. Let $N^{n}$ be a smooth connected closed $n$-dimensional manifold. It is known that every $x \in H_{n-1}\left(N ; Z_{2}\right)$ can be realized as $x=i_{*}[M]$ where $[M]$ is the $Z_{2}$ orientation class of a closed, connected (n-1)-dimensional manifold $M^{n-1}$, and $M \stackrel{i}{\rightarrow} N$ is a smooth embedding. We call such an embedded manifold a hypersurface in $N$ and say that it carries the element $x$; for convenience, a hypersurface is assumed connected. A well-known consequence of the duality theorems for manifolds asserts that $x=0$ if and only if $M$ separates $N$; that is, the complement of $i M$ consists of two components. Thus if $x=0, M$ is nullbordant. In fact by [5], two hypersurfaces which carry a given $x \in H_{n-1}\left(N ; Z_{2}\right)$ must be cobordant. This is clear if the two hypersurfaces can be made disjoint, however that is rarely possible. Note that the converse is not true: distinct elements of $H_{n-1}\left(N ; Z_{2}\right)$ may be carried by cobordant manifolds (or even the same manifold). For instance,

Received by the editors October 23, 1978 and, in revised form, September 29, 1979.

AMS (MOS) subject classifications (1970). Primary 57D40; Secondary 57D75.

Key words and phrases. Cobordism, lens space, manifold, submanifold, immersion, Stiefel-Whitney class. 
$R P^{2 n-1}$, a nullbordant manifold, carries the nonzero element in $H_{2 n-1}\left(R P^{2 n} ; Z_{2}\right)$, where $R P^{k}$ is the $k$-dimensional real projective space. In any case, if one can identify the cobordism classes of hypersurfaces carrying elements of $H_{n-1}\left(N ; Z_{2}\right)$, explicit necessary conditions are obtained for a given $(n-1)$-dimensional manifold to embed in $N$. For example, every hypersurface in $R P^{2 n}$ is nullbordant.

Further conditions can be obtained by considering the orientability of a hypersurface in $N$. Assume $N$ orientable. Note that if $M$ separates $N$ then $M$ is orientable, since $M$ is the boundary of an orientable manifold. The converse of this is true if $H_{n-1}\left(N ; Z_{2}\right)=0$, by the universal coefficient theorem. In fact this may be seen as the exact meaning that $H_{n-1}(N ; Z)=0$, for any nonzero element is carried by the inverse image of a regular value of a smooth function from $N$ to $S^{1}$, providing a nonseparating orientable hypersurface. We record these observations in the following proposition.

Proposition 1.1. Let $N$ be an orientable smooth closed $n$-dimensional manifold. Then $H_{n-1}(N ; Z)=0$ if and only if for every hypersurface $M$ in $N$ the following are equivalent:

(i) $M$ separates $N$,

(ii) $M$ is orientable.

Furthermore, $H_{n-1}\left(N ; Z_{2}\right)=0$ if and only if (i) and (ii) are true for every hypersurface $M$ in $N$.

Note that the proposition applies to any simply connected $N$, for then $H_{n-1}(N ; Z)=0$. As a corollary, if this condition holds any orientable hypersurface in $N$ is nullbordant. In general of course, orientability and cobordism type are unrelated. We exploit this perhaps unexpected relationship to obtain in Theorem A below a classification by cobordism type of hypersurfaces in lens spaces.

The following proposition is a special case of Theorem A which is immediate from the considerations above without further analysis. As noted in the first paragraph, every hypersurface in $R P^{2 n}$ is nullbordant, hence this proposition completes the classification of hypersurfaces in all real projective spaces.

Proposition 1.2. Let $M^{2 n}$ be a compact connected submanifold of $R P^{2 n+1}$. If $M$ is orientable, $M$ is nullbordant. Otherwise $M$ is cobordant to $R P^{2 n}$.

Proof. By Proposition 1.1, $M$ nonorientable implies $M$ does not separate $R P^{2 n+1}$. Therefore $M$ represents the nonzero class in $H_{2 n}\left(R P^{2 n+1} ; Z_{2}\right)$ which is also carried by $R P^{2 n}$. Thus by Thom [5], as noted in the introduction, $M$ is cobordant to $R P^{2 n}$.

An immediate consequence is the following corollary due to Bredon and Wood [1]. An elementary geometric proof along with some historical information for this result is given in [3]. (We take this opportunity to correct our citation in [3] of the result of Bredon and Wood. The Klein bottle does embed in $N \times S^{1}$ if $N=S^{2}$ : a great circle of $S^{2} \times 0$, by "time" $\theta$ in $S^{2} \times \theta$, is rotated through an angle $\theta$ (thanks to Daniel Asimov for providing this bottle).) Recall that the Klein bottle with handles is nullbordant. 
COROLlaRY. The Klein bottle does not embed in $R P^{3}$. In fact, a surface embeds in $R P^{3}$ if and only if it is orientable or of odd Euler characteristic.

Let $L_{d}^{2 n+1}$ be the quotient of $S^{2 n+1}$ defined by the coordinate-wise action in $C^{n+1}$ of the $d$ th roots of unity. Note that if $d$ is odd, $H_{2 n}\left(L_{d}^{2 n+1} ; Z_{2}\right)=0$ and hence all hypersurfaces are both orientable and nullbordant. Of course, with $d=2$ we just have Proposition 1.2.

TheOREM A. Let $M^{2 n} \stackrel{i}{\rightarrow} L_{d}^{2 n+1}$ be a hypersurface and let $d=2^{r} k$ with $k$ odd. Then if $M$ is orientable, $M$ is nullbordant. If $M$ is nonorientable, then $d$ is even and,

(i) if $r=1, M$ is cobordant to $R P^{2 n}$,

(ii) if $r>2$, and $n$ is odd, $M$ is nullbordant,

(iii) if $r>2$, and $n$ is even, $M$ is cobordant to $R P^{n} \times R P^{n}$.

The proof of Theorem $\mathbf{A}$ is given in \$2. For 3-dimensional lens spaces Bredon and Wood in [1] prove a much deeper result: the nonorientable surface of genus $s$ embeds in $L_{2 s}^{3}$ and this is in fact the minimum possible genus. They obtain as well the minimum genus of a nonorientable surface in the more general lens spaces $L^{3}(p, q)$.

The following proposition shows how to obtain nonimmersion results from nonembedding results in the case of orientable manifolds and thus extends the applicability of the previous ideas. Here $\varkappa_{*}(N)$ is the unoriented cobordism group of Conner and Floyd [2, p. 18].

Proposition 1.3. If $M^{n-1} \stackrel{f}{\rightarrow} N^{n}$ is an immersion, $M$ and $N$ smooth closed orientable manifolds, then there is an orientable $M_{0}^{n-1}$ and an embedding $M_{0} \stackrel{f_{0}}{\rightarrow} N^{n}$ where $f$ and $f_{0}$ represent the same element of $\Re_{*}(N)$.

Proof. Let $f_{*}[M] \in H_{n-1}(N ; Z) \simeq H^{1}(N ; Z)$ be represented by a smooth map $N \rightarrow S^{1}$. Let $M_{0}$ be the inverse image of a regular value and $f_{0}$ the inclusion into $N$. Then $f$ and $f_{0}$ represent the same element of $H_{n-1}(N ; Z)$ hence of $H_{n-1}(N ; Z)$. Then since the normal bundles are trivial, it is easy to see that they have equal Whitney numbers in the sense of $[2, \S 17]$.

COROLlaRY. If $M^{2 n}$ is orientable and immerses in $L_{d}^{2 n+1}$, then $M$ is nullbordant.

In this manner one can see for example that there are no codimension one immersions of complex projective spaces $C P^{2 n}, n>1$, into lens spaces.

2. Corresponding to the various subgroups of the $d$ th roots of unity we have the lattice of covering spaces

$$
S^{2 n+1} \rightarrow \cdots \rightarrow L_{d_{1}}^{2 n+1} \rightarrow \cdots \rightarrow L_{d}^{2 n+1}
$$

where each $d_{i}$ is a divisor of $d$. Thus if $d$ is even, $R P^{2 n+1}$ is a covering space intermediate between $L_{d}^{2 n+1}$ and $S^{2 n+1}$. The next proposition together with the results of $\$ 1$ will then establish parts (i) and (ii) of Theorem A: lift $M$ back to its 
preimage $\bar{M}$ in (i) $S^{2 n+1}$ or (ii) $R P^{2 n+1}$ respectively, and apply the proposition. Of course (i) also follows from Proposition 1.1.

Proposmion 2.1. If $\bar{M}^{p} \stackrel{M}{\rightarrow}$ is an odd degree covering projection, then $\bar{M}$ is cobordant to $M$.

Proof. Since $p_{*}[\bar{M}]=[M]$ in homology with $Z_{2}$ coefficients, it follows immediately from naturality that the Stiefel-Whitney numbers are equal.

From here on we assume $d=2^{r}$ with $r>2$, for if $d=2^{r} k$ and $k$ is odd, we may lift $M$ in $L_{d}^{2 n+1}$ back to $\bar{M}$ in $L_{2^{r}}^{2 n+1}$ and apply Proposition 2.1 to the $k$-fold cover of $M$ by $\bar{M}$. If $\bar{M}$ is not connected, choose some component of $\bar{M}$ which is an odd degree cover of $M$. Note that the case $r=1$ is given in Proposition 1.2.

Proposition 2.2 below gives the $Z_{2}$ cohomology ring of $L_{2^{\prime}}^{2 n+1}$. The additive structure of course is well known to be $Z_{2}$ in all degrees.

Proposition 2.2. $H^{*}\left(L_{2^{r}}^{2 n+1} ; Z_{2}\right), r>2$, is $Z_{2}$ in each degree generated by $u, a$ of degrees one and two respectively, where $u^{2}=0$. Furthermore, the total Stiefel-Whitney class of $L_{2^{\prime}}^{2 n+1}$ is $W(L)=(1+a)^{n+1}$.

Proor. This result follows by induction on $r$ from the following observations. Let $\bar{N} \stackrel{p}{\rightarrow} N$ be a connected 2 -fold cover where the $Z_{2}$ cohomology of $\bar{N}$ and of $N$ is (additively) $Z_{2}$ in every degree. Let $u \in H^{1}\left(N ; Z_{2}\right)$ be the nonzero element. Then the Gysin sequence for $p$ is

$$
\begin{aligned}
H^{0}(N) & \stackrel{\approx}{\rightarrow} H^{1}(N) \stackrel{p^{*}}{\rightarrow} \cdots \\
1 & \mapsto u \\
\ldots & \rightarrow H^{j}(N) \stackrel{p^{*}}{\rightarrow} H^{j}(\bar{N}) \rightarrow H^{j}(N) \stackrel{\cup u}{\rightarrow} H^{j+1}(N) \rightarrow \cdots
\end{aligned}
$$

Thus $p^{*}$ is zero in odd degrees and an isomorphism in even degrees. Further, if $q^{*}(x)=x \cup u, q^{*}$ is also zero in odd degrees and an isomorphism in even degrees. In particular, $u^{2}=0$ and the odd degree Stiefel-Whitney classes of $\bar{N}$ are zero by naturality. Thus considering the composition of coverings

$$
R P^{2 n+1} \rightarrow L_{4} \rightarrow \cdots \rightarrow L_{2^{r}} \rightarrow L_{2^{r+1}}
$$

we deduce that $w_{j}\left(L_{2^{\prime}}\right)=w_{j}\left(R P^{2 n+1}\right)$ because $p^{*}$ is an isomorphism for $j$ even and because both classes are zero for $j$ odd.

Let $l=2^{j}$ be the highest power of 2 which divides $n$. Then $W(L)=(1+a)^{n}=$ $\left(1+a^{l}\right)^{\text {odd }}=1+a^{l}+\cdots(\bmod 2)$. Hence we have

LEMMa 2.1. Let $\bar{w}_{k}$ denote the kth Stiefel-Whitney class of $L_{2^{r}}^{2 n+1}$. If $\bar{w}_{k} \neq 0$, then $l=2^{j}$ divides $k$ and $\bar{w}_{k}=\bar{w}_{l}^{m}$ where $\operatorname{lm}=k$.

LEMMA 2.2. Let $w_{k}$ be the kth Stiefel-Whitney class of a nonorientable manifold $M^{2 n}$ and let $u \in H^{1}\left(L_{2^{r}}^{2 n+1} ; Z_{2}\right)$ be the nonzero element. Let $M \stackrel{i}{\rightarrow} L_{2^{r}}^{2 n+1}$ be an embedding. Then

(i) $w_{k}=i^{*}\left(\bar{w}_{k-1} u\right)$ if $k$ is odd,

(ii) $w_{k}=i^{*}\left(\bar{w}_{k}\right)$ if $k$ is even. 
Proof. Since $M$ is nonorientable, $i_{*}[M] \neq 0$ in $Z_{2}$ homology. The class $w_{1}(\nu(i))$ $=i^{*} u$ is Poincaré dual to $i_{*}[M]$. Thus $i^{*} W(L)=W(M) \cup\left(1+i^{*} u\right)$. Since $u^{2}=$ 0 , this gives $W(M)=i^{*}(W(L) \cup(1+u))$. Hence, since $\bar{w}_{k}=0$ for $k$ odd,

$$
w_{k}=i^{*}\left(\bar{w}_{k}+\bar{w}_{k-1} u\right)= \begin{cases}i^{*} \bar{w}_{k}, & k \text { even }, \\ i^{*}\left(\bar{w}_{k-1} u\right), & k \text { odd }\end{cases}
$$

We now conclude the proof of Theorem $A$.

Proof of Theorem A (iii). Assume $n$ odd. Consider a nonzero Stiefel-Whitney number of $M$

$$
\begin{aligned}
0 & \neq\left\langle w_{1}^{r_{1}} \cdots w_{s}^{r_{s}},[M]\right\rangle=\left\langle i^{*}\left(\alpha u^{r_{0}}\right),[M]\right\rangle \\
& =\left\langle\alpha u^{r_{0}}, u \cap[L]\right\rangle=\left\langle\alpha u^{r_{0}+1},[L]\right\rangle .
\end{aligned}
$$

Here $\alpha$ is a monomial in even degree $\bar{w}$ 's given by Lemma $2.2(i)$. Since $u^{2}=0, r_{0}=$ 0 , thus all $w_{k}$ 's here are of even degree. Thus this Stiefel-Whitney number is $\left\langle\bar{w}_{2}^{m}, u \cap[L]\right\rangle$ where $\bar{w}_{2^{\prime}}$ is the first nonzero Stiefel-Whitney class of $L$. Thus $m 2^{j}=2 n$ so $m 2^{j-1}$ is odd (since $n$ is odd). But $j>1$ since when $n$ is odd $\bar{w}_{2}=0$ $\left(\bar{w}_{2}=\left(\begin{array}{c}n_{1}^{+1} \\ 1\end{array}\right)\right)$. This is a contradiction, hence all Stiefel-Whitney numbers of $M$ are zero.

Proof of Theorem A (iv). Assume $\boldsymbol{n}$ even. In this case as well, any monomial with an odd degree Stiefel-Whitney class will yield a zero Stiefel-Whitney number of $M$ because of the introduction of $u^{2}=0$. Let us now establish this property for the Stiefel-Whitney numbers of $R P^{n} \times R P^{n}$.

Let $\bar{\omega}_{k}, \omega_{k}$ be the $k$ th Stiefel-Whitney classes of $R P^{n} \times R P^{n}$ and $R P^{n}$ respectively. Recall that $\bar{\omega}_{k}=\Sigma_{j=0}^{k} \omega_{j} \times \omega_{k-j}$ and $\omega_{k-j} \times \omega_{j}$ are either both zero or both nonzero. Thus if $k$ is odd, a Stiefel-Whitney number having a monomial with $\bar{\omega}_{k}$ becomes twice the corresponding Stiefel-Whitney number with $\bar{\omega}_{k}$ replaced by $\sum_{j=0}^{(k-1) / 2} \omega_{j} \times \omega_{k-j}$. Thus any Stiefel-Whitney number of $R P^{n} \times R P^{n}$ with an odd degree Stiefel-Whitney class will be zero. A similar, somewhat more involved argument will establish the equivalence of statements (1) and (2) below.

We complete the proof of Theorem A (iv) by noting successively that the statements (1)-(7) below are equivalent. For this we need to establish that $i^{*}$ is one to one in even degrees. To see this note $\left(i^{*} a\right)^{n} \neq 0$ since $\left\langle i^{*} a^{n},[M]\right\rangle=\left\langle a^{n} u,[L]\right\rangle$ $\neq 0$ by Proposition 2.2. The following chain of equivalent statements completes the demonstration that $M$ and $R P^{n} \times R P^{n}$ have the same Stiefel-Whitney numbers.

(1) $\left\langle\bar{\omega}_{2}^{r_{1}} \cdots \bar{\omega}_{2 s}^{r_{s}},\left[R P^{n} \times R P^{n}\right]\right\rangle \neq 0$,

(2) $\left\langle\left(\omega_{1} \times \omega_{1}\right)^{r_{1}} \cdots\left(\omega_{s} \times \omega_{s}\right)^{r_{s}},\left[R P^{n} \times R P^{n}\right]\right\rangle \neq 0$,

(3) $\omega_{i} \times \omega_{i} \neq 0$ for all $i$,

(4) $\omega_{i} \neq 0$ for all $i$,

(5) $\bar{w}_{2 i}^{r_{i}} \neq 0$ for all $i$ (a direct computation),

(6) $w_{r_{1}}^{r_{i}} \neq 0$ for all $i$ (Lemma 2.3 since $i^{*}$ is 1-1),

(7) $\left\langle w_{2}^{r_{1}} \cdots w_{2 s}^{r_{s}},[M]\right\rangle \neq 0$ (Lemma 2.2). 


\section{BIBLIOGRAPHY}

1. G. E. Bredon and J. W. Wood, Non-orientable surfaces in orientable 3-manifolds, Invent. Math. 7 (1969), 83-110.

2. P. E. Conner and E. E. Floyd, Differentiable periodic maps, Academic Press, New York, 1964.

3. J. H. C. Creighton, An elementary proof of the classification of surfaces in real projective 3-space, Proc. Amer. Math. Soc 72 (1978), 191-192.

4. John W. Milnor and James D. Stasheff, Characteristic classes, Princeton Univ. Press., Princeton, N. J., 1974.

5. R. Thom, Quelques propriétés globales des variétés differentiables, Comment. Math. Helv. 28 (1954), 17-86.

Current address: Sandeepany West, P. O. Box 9, Piercy, California 95467 